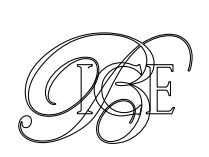

\title{
COMPETENCIA Y REGULACIÓN EN LOS MERCADOS DE COMUNICACIONES ELECTRÓNICAS: BANDA ANCHA Y DESPLIEGUES DE NUEVA GENERACIÓN
}

La calidad de las infraestructuras de telecomunicaciones es un factor clave para aumentar la competitividad e impulsar la transformación digital del país. En las redes fijas de telecomunicaciones, la arquitectura que permite las velocidades de transmisión más elevadas es la fibra hasta el hogar (Fiber-To-The-Home, FTTH). España es un caso de éxito en los despliegues de fibra hasta el hogar, realizados además por varios operadores. La cobertura de redes FTTH alcanzaba el $77 \%$ en junio de 2018 y España es el país de Europa con mayor número de hogares conectados mediante esta tecnología. El presente artículo analiza los factores que han contribuido a este éxito. Para ello, se expondrá el desarrollo del mercado de banda ancha en los últimos años, explicando la dinámica competitiva de este mercado y el impacto positivo de las medidas adoptadas por la CMT y la CNMC ${ }^{1}$, en especial la imposición en 2009 del acceso a la infraestructura civil de Telefónica complementada con las obligaciones de acceso simétricas en el interior de los edificios y la evolución del marco regulatorio con el análisis geográfico efectuado en 2016.

Palabras clave: FTTH, conductos, CMT, CNMC, banda ancha, conectividad, telecomunicaciones, regulación.

Clasificación JEL: L43, L96.

\section{Introducción}

El fomento de la inversión en redes de muy alta capacidad es cada vez más importante para la competitividad y el desarrollo económico. Por ello, la Comisión Europea ha establecido unos ambiciosos objetivos de conectividad para 2025.

\footnotetext{
* Subdirector de Análisis de Mercados de Comunicaciones Electrónicas. Comisión Nacional de los Mercados y la Competencia. Versión de abril de 2019.

DOI: https://doi.org/10.32796/bice.2019.3111.6819
}

España se encuentra muy bien situada para alcanzar dichos objetivos debido a los relevantes despliegues de redes de nueva generación (Next Generation Access, NGA) realizados por varios operadores.

El presente artículo analiza los factores que han contribuido a este éxito. Para ello, se expondrá el desarrollo del mercado de banda ancha en los últimos años, explicando la $\triangleright$

\footnotetext{
La Comisión del Mercado de las Telecomunicaciones (CMT) era el regulador de las telecomunicaciones hasta su integración en 2013 dentro de la actual Comisión Nacional de los Mercados y de la Competencia (CMNC).
} 


\section{Objetivos de conectividad establecidos por la Comisión Europea para 2025}

1. Todos los motores socioeconómicos importantes, como los centros escolares, las universidades, los centros de investigación, los nudos de transporte, todos los proveedores de servicios públicos, como los hospitales y las Administraciones, y las empresas que dependen de las tecnologías digitales deben tener acceso a una conectividad de velocidad extremadamente alta que permita a los usuarios descargar/cargar 1 gigabyte de datos por segundo.

2. Todos los hogares europeos, rurales o urbanos, deben tener acceso a una conectividad que ofrezca una velocidad de descarga de al menos $100 \mathrm{Mbps}$, mejorable hasta el Gbps.

3. Todas las zonas urbanas, así como las principales carreteras y ferrocarriles, deben tener cobertura ininterrumpida de 5G, la quinta generación de sistemas de comunicaciones inalámbricas. Como objetivo intermedio, $5 \mathrm{G}$ debe estar comercialmente disponible al menos en una de las principales ciudades de cada Estado miembro de la UE en 2020.

En relación con el tercer objetivo, cabe señalar que la futura generación de tecnología móvil (5G) necesita que se conecten multitud de estaciones base en todo el territorio con fibra óptica. Por este motivo, un elevado despliegue de fibra óptica también facilita el futuro despliegue de la tecnología $5 \mathrm{G}$, ya que es más fácil conectar las estaciones base con fibra óptica.

dinámica competitiva de este mercado y la situación de España respecto al resto de Europa. A continuación se analizará el impacto de las medidas regulatorias adoptadas por la CMT y la CNMC en la situación actual. Por último, se explicará la relación entre estas medidas y el Código de las Comunicaciones Electrónicas aprobado el 11 de diciembre de 2018 en el Parlamento Europeo.

\subsection{La banda ancha y las redes de nueva generación}

Los servicios de banda ancha fija se han prestado tradicionalmente mediante tecnología $\mathrm{xDSL}^{2}$ sobre accesos de cobre. Estas líneas, salvo excepciones, no permiten prestar velocidades por encima de $30 \mathrm{Mbit} / \mathrm{s}$.

\footnotetext{
2 La familia xDSL (Digital Subscriber Loop) engloba diversas tecnologías que se prestan por el par telefónico de cobre. La tecnología más conocida es ADSL, caracterizada por la asimetría de las conexiones (la velocidad de descarga es superior a la de subida).
}

Las redes de nueva generación (Next Generation Access, NGA) se definen como aquéllas que permiten prestar velocidades por encima de $30 \mathrm{Mbit} / \mathrm{s}$. En las redes NGA, la fibra óptica es fundamental, debido a que es un medio de transmisión óptimo con reducida atenuación y resistencia frente a las interferencias, por lo que permite velocidades muy superiores al cobre.

La primera red de nueva generación fue la red de cable con la tecnología HFC (Híbrido Fibra Coaxial). La terminología HFC indica que la red de acceso tiene dos tramos diferenciados, uno con fibra óptica y otro con cable coaxial: la central del operador se conecta con diversos puntos intermedios (nodos) mediante fibra óptica y desde cada uno de estos nodos parte una red de distribución de cable coaxial que llega hasta cada uno de los hogares. Esta tecnología permite proporcionar velocidades de hasta $1 \mathrm{Gbps}$, dependiendo de diversos factores.

Por su parte, las redes de acceso de cobre también pueden evolucionar hacia las redes $D$ 
NGA. Para ello, hay que sustituir la conexión de cobre por fibra óptica. La familia FTTx (del inglés Fiber to the $x$ ) cubre las diferentes opciones en las que la fibra óptica sustituye parcial o totalmente el par de cobre del bucle de acceso que llega a los hogares. El acrónimo FTTx se origina como generalización de las distintas configuraciones existentes (FTTN, FTTC, FTTB, FTTH...), diferenciándose por la última letra que indica dónde termina la fibra (Node/nodo, Cabinet/armario, Building/edificio, Home/hogar...). Por ejemplo, en FTTN se sustituye el cobre por la fibra óptica en el primer tramo de la red de acceso — desde la central del operador a un nodo que tiene una cobertura de cientos de viviendas-, pero se mantiene el acceso de cobre en el tramo final que va desde el nodo hasta cada uno de los hogares ${ }^{3}$.

Cuanto mayor es el tramo cubierto por fibra óptica, mayores son las velocidades alcanzables. Por tanto, FTTH es la arquitectura NGA que permite las mayores velocidades, pues la fibra óptica llega hasta el domicilio del cliente y el par de cobre desaparece completamente ${ }^{4}$.

\section{El mercado de banda ancha en España}

Las primeras ofertas de banda ancha comenzaron a comercializarse en España en 1999 con la tecnología ADSL y con una velocidad de $256 \mathrm{~Kb} / \mathrm{s}$. Rápidamente surgieron los empaquetamientos de banda ancha fija con

\footnotetext{
3 Nótese que la arquitectura FTTN es muy similar a HFC, con la diferencia de que el tramo final es un par de cobre en FTTN y un cable coaxial en HFC.

4 La tecnología FTTH también tiene otras ventajas, como una disminución del consumo eléctrico del operador, de las centrales locales necesarias, de la ocupación de los conductos y de los costes de mantenimiento.
}

tarifas planas de voz. A su vez, los operadores de cable comenzaron a desplegar redes HFC e incluían en su oferta, además de banda ancha y telefonía, televisión de pago. Los competidores del mercado eran Telefónica, los operadores que hacían uso de servicios mayoristas sobre la red de cobre $^{5}$ y los operadores de cable. Durante varios años, los servicios de comunicaciones fijas y móviles se comercializaron por separado.

Sin embargo, en octubre de 2012 Telefónica lanzó Movistar Fusión, un producto convergente que incluía telefonía fija, banda ancha fija, telefonía móvil y banda ancha móvil. Este producto representaba un ahorro cercano al $40 \%$ respecto de la compra por separado de los servicios fijos y móviles, por lo que tuvo un éxito inmediato y la competencia lanzó productos convergentes similares. Actualmente, más del $80 \%$ de líneas de banda ancha fija se comercializan empaquetadas en una oferta convergente con una o más líneas móviles.

Asimismo, en 2012 las únicas redes NGA con niveles de cobertura elevados eran las de los operadores de cable. ONO contaba con la red HFC más extensa a nivel nacional y tres operadores de cable regionales (Euskaltel en el País Vasco, Telecable en Asturias y R en Galicia) contaban con una cobertura muy relevante en sus territorios. La competencia de la infraestructura alternativa de la red de cable sin duda influyó en la decisión de Telefónica de evolucionar su red de cobre xDSL hacia la arquitectura de red NGA que permitía las mayores velocidades, la fibra hasta el hogar.

Estos dos factores, la convergencia fijo-móvil y la apuesta clara de Telefónica por un $\triangleright$

\footnotetext{
5 El servicio mayorista más relevante en redes de cobre ha sido la desagregación del bucle de abonado de Telefónica. Los operadores alternativos se coubican en las centrales locales de Telefónica y acceden directamente al bucle de abonado del cliente final.
} 
despliegue de red FTTH, explican, al menos en parte, dos fusiones muy relevantes en las que un operador predominantemente móvil adquirió un operador fijo con una red NGA.

En julio de 2014, Vodafone adquirió ONO 6 (entonces disponía de la mayor red NGA) y en mayo del año siguiente Orange adquirió Jazztel $^{7}$ (que contaba con una notable red FTTH gracias a un acuerdo de coinversión firmado en 2013 con Telefónica).

Si bien las operaciones anteriores permitían tanto a Vodafone como a Orange competir mejor frente a Telefónica en un entorno convergente, el mercado se redujo de cinco a tres operadores nacionales. Por este motivo, la Comisión Europea exigió a Orange la presentación de unos compromisos para aprobar la adquisición de Jazztel. Estos compromisos tenían como objetivo la creación de un nuevo cuarto operador convergente nacional que compensara la pérdida de presión competitiva derivada de la desaparición de Jazztel. El operador que hizo uso de estos compromisos fue MásMóvil —hasta entonces un operador móvil virtual de tamaño reducido-, que alcanzó una serie de acuerdos con Orange. Además de estos acuerdos, MásMóvil adquirió diversos operadores, entre los que destaca Yoigo, el cuarto operador móvil. Tras estas operaciones, Yoigo comenzó a competir con notable éxito en el segmento de ofertas convergentes fijo-móvil sin televisión.

Asimismo, en abril de 2015 Telefónica (líder del mercado de banda ancha) adquirió DTS (líder del mercado de televisión de pago). Con esta operación, Telefónica reforzaba el tercer pilar de su estrategia competitiva:

\footnotetext{
6 Caso M.7231 VODAFONE/ONO (http://ec.europa.eu/competition/ elojade/isef/case_details.cfm?proc_code=2_M_7231).

7 Caso M.7421 ORANGE/JAZZTEL (http://ec.europa.eu/competition/ elojade/isef/case_details.cfm?proc_code=2_M_7421).
}

convergencia, despliegues de red FTTH y apuesta por los contenidos de televisión de pago. Esta operación fue supervisada por la CNMC, que también exigió la presentación de una serie de compromisos para su aprobación ${ }^{8}$. Uno de los objetivos de los compromisos fue evitar que Telefónica se apalancara en su posición de liderazgo en la televisión de pago para disminuir la competencia en el mercado de banda ancha.

Por ello, desde 2015 hasta el presente, el mercado de banda ancha plantea dinámicas competitivas crecientemente complejas:

- Tres grandes operadores (Telefónica, Vodafone y Orange) con red fija y móvil compiten a nivel nacional con ofertas integradas que incluyen fijo, móvil y televisión de pago. En este segmento se ha observado con mayor fuerza la práctica denominada «más-por-más» seguida por los tres operadores: aumentos de precios justificados por los operadores en las mayores prestaciones (más TV, más banda ancha fija y más móvil a cambio de mayor precio, pero al cliente no se le da opción de rechazar la mejora). Esta estrategia se combina con un creciente esfuerzo promocional para atraer y retener clientes.

- En el segmento de las ofertas integradas sin televisión de pago, MásMóvil ha irrumpido con mucho éxito con una estrategia de precios atractivos, alcanzando en apenas tres años una cuota de mercado de banda ancha cercana al $7 \%$. Los tres grandes operadores han reaccionado lanzando segundas $\triangleright$

8 Caso C/0612/14: TELEFÓNICA/DTS (https://www.cnmc.es/expedientes/ c061214). 


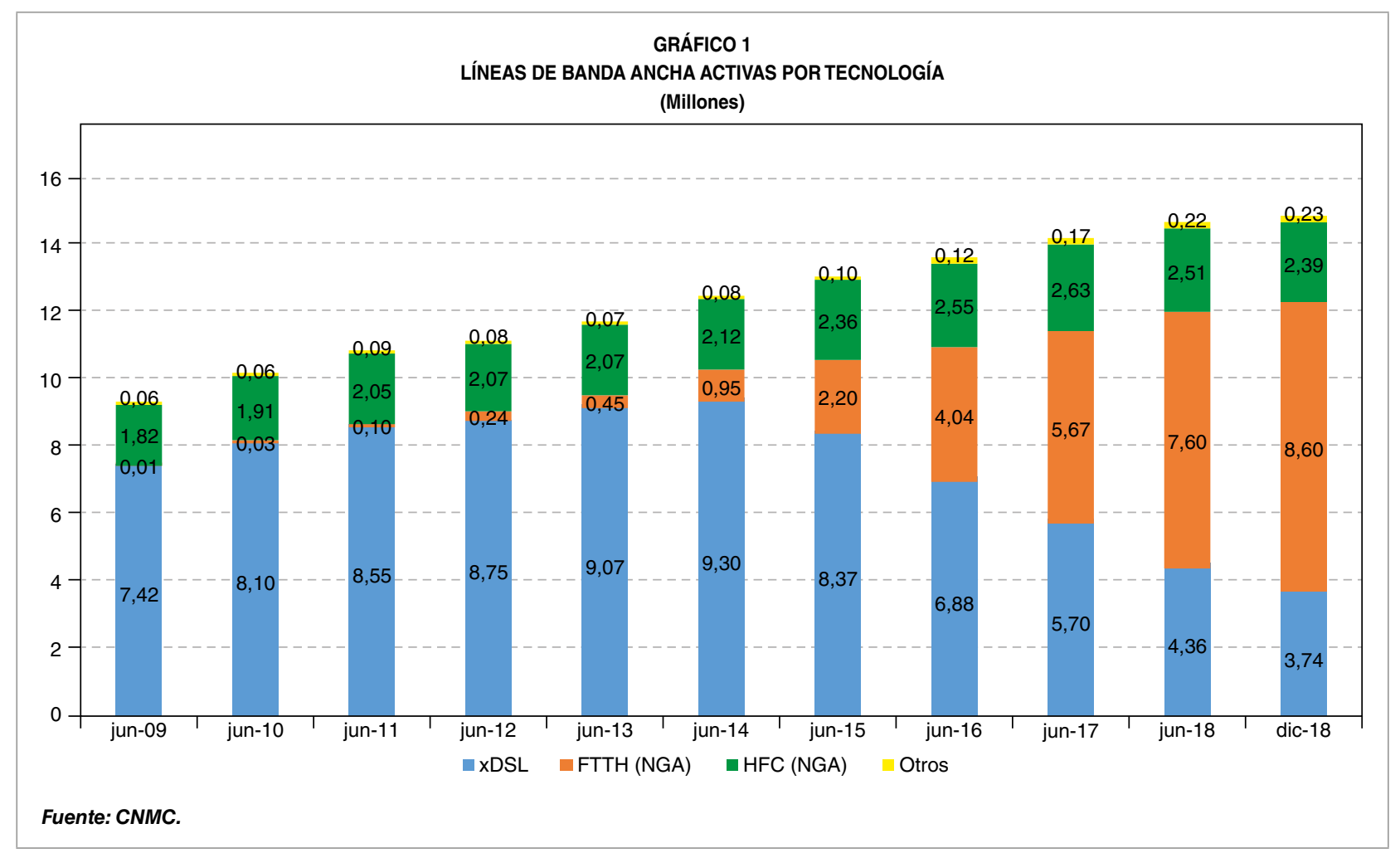

marcas $^{9}$ con ofertas competitivas a precios más reducidos ${ }^{10}$.

\subsection{Evolución del mercado de banda ancha}

El número de líneas de banda ancha activas $^{11}$ ha pasado de 9,3 millones en junio de 2009 a 15,0 millones en diciembre de 2018, lo que supone un crecimiento de un $61 \%$ en nueve años y medio.

Además del crecimiento en el número de líneas, en el mercado español se observa una intensa migración de las conexiones de cobre (xDSL) a redes de nueva generación (NGA), en especial de fibra hasta el hogar (FTTH).

9 Movistar: Tuenti y O2, Vodafone: Lowi y Vodafone Bit, Orange: Jazztel y Amena).

10 Conocidas como de ofertas de «bajo coste» o también «value-for-money».

11 Es decir, las líneas en servicio contratadas por un hogar, autónomo o una empresa.
El número de líneas de nueva generación ha pasado de 1,8 millones en 2009 - casi todas HFC- a 11 millones en 2018 -el $80 \%$ de ellas FTTH- La tecnología xDSL, predominante hasta 2016, ahora representa menos del $25 \%$ de todas las líneas.

\subsection{Despliegues de nueva generación}

El mercado español de banda ancha fija destaca a nivel europeo por el elevado nivel de cobertura y por contar con despliegues de redes de nueva generación por parte de diversos operadores: Telefónica, Vodafone, Orange, MásMóvil, el Grupo Euskaltel y también por multitud de pequeños operadores locales.

El Gráfico 2 muestra la evolución en el número de unidades inmobiliarias (UU II $\left.{ }^{12}\right) \triangleright$

12 Una unidad inmobiliaria es una ubicación donde potencialmente se puede instalar una acometida de fibra. Cubre hogares, viviendas desocupadas y locales comerciales. 


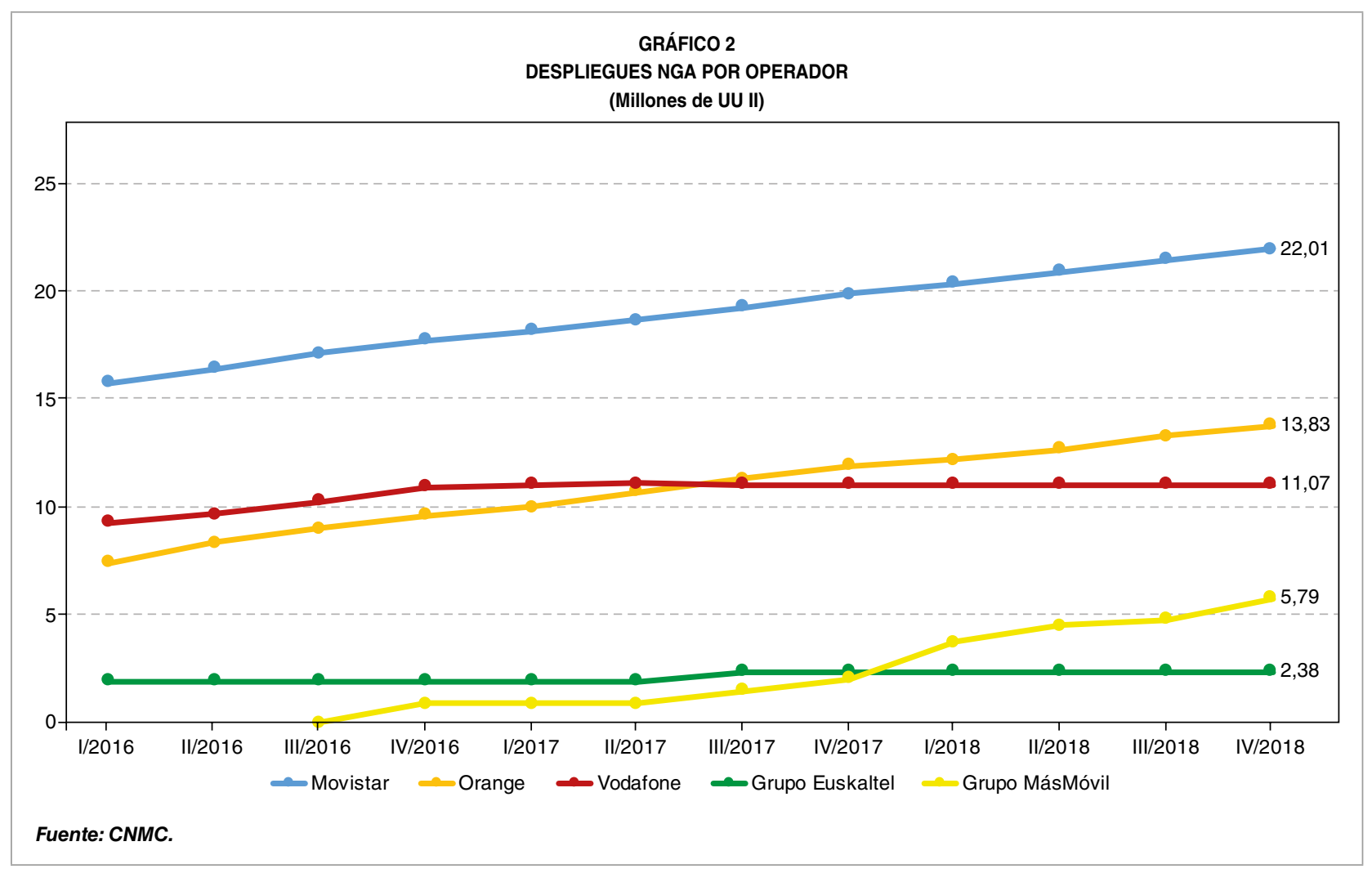

pasadas con fibra en los últimos años, por los cuatro principales operadores de ámbito nacional, así como por el Grupo Euskaltel.

Con 22 millones de unidades inmobiliarias, la cobertura actual de la red de fibra de Telefónica es muy elevada, cercana al $80 \%$. La cobertura de red propia de Orange y Vodafone es inferior (14 y 11 millones respectivamente), pero gracias a la utilización de los servicios mayoristas de Telefónica, la cobertura total de Orange y Vodafone ${ }^{13}$ es similar a la de Telefónica, en torno a 22 millones de UU II. Por su parte, la cobertura de red propia de MásMóvil es la más reducida de los cuatro (5,8 millones), pero si se incluye el uso de servicios mayoristas, la cobertura total es también muy relevante $(15,2 \text { millones })^{14}$.

\footnotetext{
13 La obtenida mediante red propia más la obtenida mediante el acceso mayorista de otro operador.

14 MásMóvil utiliza principalmente los servicios mayoristas de Orange y, en menor medida, de Telefónica y Vodafone.
}

Finalmente, cabe mencionar que estos planes de despliegue continúan: para 2020, Telefónica ha anunciado que espera alcanzar una cobertura de aproximadamente 25 millones de unidades inmobiliarias y Orange, de 16 millones. Es decir, es previsible que la cobertura de las redes de nueva generación de alta capacidad supere el $90 \%$ en un plazo de tiempo relativamente breve.

\section{Comparativa europea}

"Spain now has the most extensive fibre-to-home network in Europe». The Economist, 2/4/2016

España es uno de los países con mayores despliegues de redes de fibra hasta el hogar, con niveles de despliegue comparables a los existentes en países como Corea y $\triangleright$ 
Japón. Los últimos datos de cobertura publicados por la Secretaría de Estado para el Avance Digital señalan que la cobertura de redes de nueva generación, capaz de soportar velocidades por encima de $30 \mathrm{Mb} / \mathrm{s}$ ascendía al $85 \%$, mientras que la cobertura de las redes FTTH ascendía al $77 \%$. Estos datos corresponden al 30 de junio de 2018; la cobertura a diciembre de 2018 de redes FTTH se situaba próxima al $80 \%$.

España cuenta con las dos redes FTTH más extensas de Europa: la de Telefónica, con 21,3 millones de unidades inmobiliarias pasadas y la de Orange España, con 13,8 millones ${ }^{15}$.

El despliegue de estas redes de fibra viene acompañado por una adopción masiva de los servicios de banda ancha sobre FTTH por parte de los hogares españoles. El Gráfico 3 muestra la penetración de las tecnologías FTTB/ $\mathrm{FTTH}^{16}$ en Europa. España es el tercer país si se tienen en cuenta ambos tipos de tecnología, pero es el líder claro en cuanto a penetración de la tecnología FTTH. Así, mientras que el $44 \%$ de los hogares españoles tienen una conexión de banda ancha FTTH, la penetración media en la UE se sitúa en torno al 10\%.

\section{El impacto de la regulación en los despliegues de fibra óptica}

El despliegue de fibra se ha visto favorecido por obligaciones regulatorias que promueven

\footnotetext{
15 Datos a 31 de diciembre de 2018. Es interesante destacar que la red de Orange España (13,8 millones) es más extensa que la de Orange Francia (11,8 millones), a pesar de que Orange es el operador histórico en el país vecino y que la población de Francia es un $40 \%$ superior a la de España.

16 Como se ha explicado en el apartado 1.1, en FTTB la fibra llega hasta la entrada del edificio, pero el tramo final que conecta cada hogar en el interior puede continuar siendo el par de cobre. Por el contrario, con FTTH la fibra óptica llega hasta el hogar y el par de cobre desaparece completamente.
}

la inversión en infraestructuras ${ }^{17}$. A continuación se explican las medidas adoptadas por la CMT en 2009, que fueron fundamentales para fomentar los despliegues de red FTTH. Posteriormente, se explica la revisión realizada por la CNMC en 2016, cuyo objetivo era lograr el balance adecuado entre fomentar la inversión eficiente y asegurar el mantenimiento de una competencia sostenible a la luz de los despliegues realizados por los diferentes operadores.

\subsection{El marco regulatorio adoptado por la CMT 2009}

En 2009, la CMT adoptó dos decisiones muy importantes para el desarrollo de la competencia y el despliegue de redes de nueva generación: la revisión de los mercados de banda ancha y la decisión sobre obligaciones de acceso simétrico en el interior de los edificios.

En 2009 ya existía una red de nueva generación, la red de cable con la tecnología HFC, si bien la mayoría de conexiones de banda ancha se prestaba mediante líneas de cobre con la tecnología xDSL. La CMT era consciente de que era posible y deseable que la red de cobre también evolucionara tecnológicamente hacia arquitecturas NGA. Para ello, debía acercarse la fibra óptica hasta el domicilio del cliente.

La obra civil (canalizaciones, conductos, arquetas, postes) supone el coste más $\triangleright$

17 Los planes de ayudas públicas, en especial el Programa de Extensión de la Banda Ancha de Nueva Generación (PEBA-NG) de la Secretaría de Estado para el Avance Digital (SEAD), juegan un papel muy relevante para que aumente el nivel de cobertura, en especial en zonas rurales donde no llegaría la inversión privada. Según la SEAD, las ayudas concedidas en el periodo 2013-2017 han permitido llevar la fibra a 3,6 millones de unidades inmobiliarias. El plan PEBA-NG actual tiene como objetivo que el $93,45 \%$ de la población tenga acceso a redes de banda ancha con velocidades al menos de $100 \mathrm{Mb} / \mathrm{s}$ a finales de 2021. En todo caso, el objeto del presente artículo es analizar el impacto de la regulación en los despliegues de los operadores realizados mediante la inversión privada. 


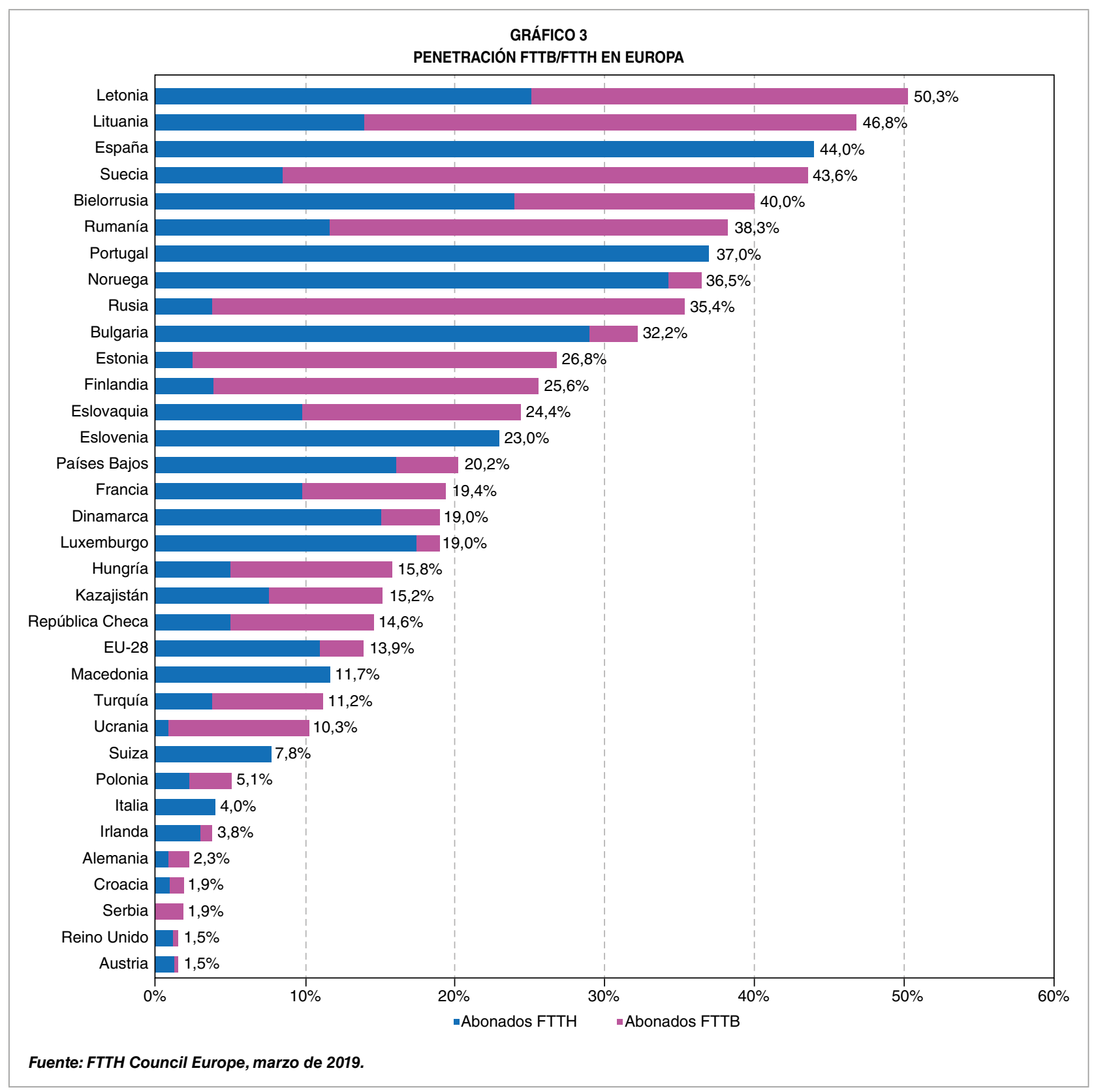

relevante del despliegue de una red de banda ancha de nueva generación ${ }^{18}$. Telefónica ya disponía de esta infraestructura legada de la red de cobre, por lo que la CMT adoptó una decisión muy relevante: obligar a Telefónica a ofrecer acceso mayorista sobre esta infraestructura

18 Distintos estudios estiman que la obra civil supone entre el $60 \%$ y el $80 \%$ de los costes de despliegue de la red de acceso. La OCDE estima un $70 \%$ (ver «Public rights of way for fiber deployment to the home", abril, 2008, disponible en www.oecd.org/dataoecd/49/9/40390753.pdf). para que los operadores alternativos pudieran desplegar sus propias redes de fibra óptica.

Asimismo, la CMT estableció un límite de $30 \mathrm{Mb} / \mathrm{s}$ para los servicios de acceso mayorista de Telefónica. El mensaje regulatorio estaba claro: para competir en servicios de banda ancha de nueva generación los operadores debían desplegar su propia red de fibra, para lo que contaban con la notable facilidad de acceder a la infraestructura civil de Telefónica. 


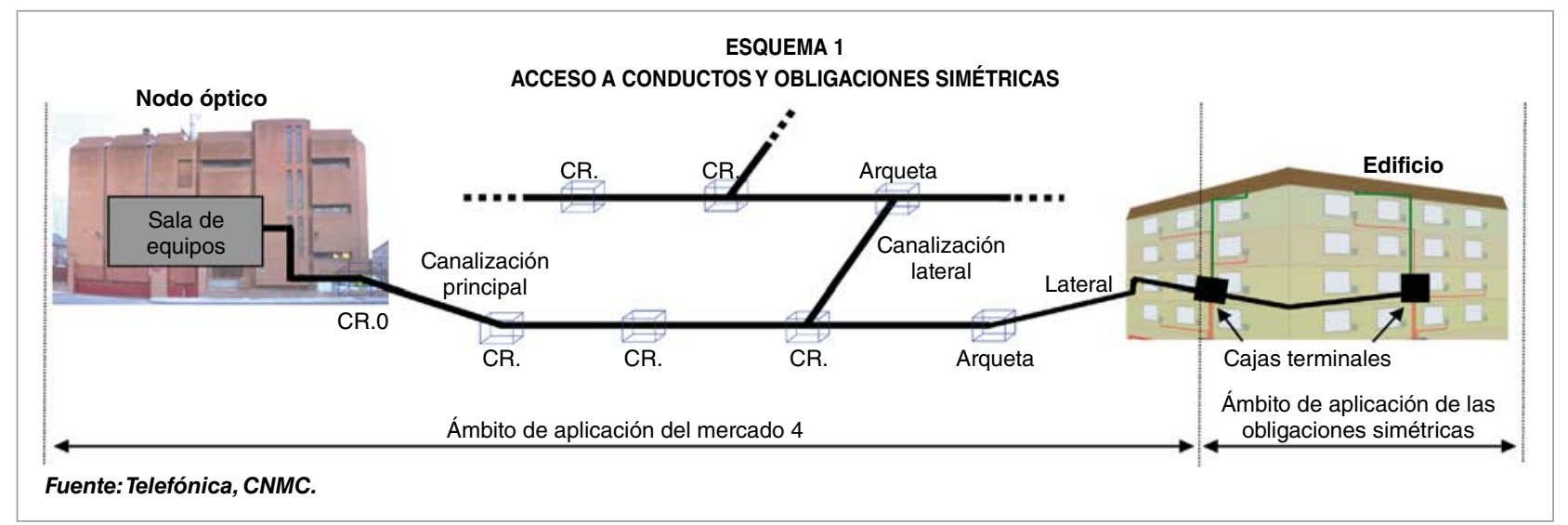

Por último, los despliegues FTTH cuentan con una problemática adicional frente a otras redes NGA en las que el último tramo todavía se presta mediante cobre, como VDSL, usada intensivamente, por ejemplo, en Alemania. Para llevar la fibra hasta el hogar, los operadores deben realizar una instalación en el interior de los edificios. Sin ningún tipo de regulación, el primer operador que despliega su red de fibra en el interior de un edificio cuenta con una relevante ventaja, ya que, bien por falta de espacio o por las reticencias de los vecinos, podría no ser viable instalar una segunda o tercera red de fibra en el edificio. Por ello, la CMT adoptó una segunda decisión, complementaria a la regulación de mercados de banda ancha, por la que obligaba a todos los operadores, no solo a Telefónica, a ofrecer acceso a la red de fibra desplegada en el interior de un edificio a precios razonables. Es decir, el primer operador que despliega una infraestructura de fibra óptica está obligado a compartirla con el resto de operadores ${ }^{19}$.

Estas medidas han sido muy importantes en el desarrollo de las redes de fibra que se han desplegado en los últimos años. Además, el acceso a los conductos y la obligación simétrica

19 La normativa de Infraestructura Común de Telecomunicaciones, adoptada en 1998, y sus sucesivos desarrollos también han facilitado en gran medida el despliegue de varias redes en el interior de los edificios construidos a partir de 1998. de acceso a las infraestructuras de fibra en los edificios permiten tener las ventajas de la competencia entre varias redes de fibra, evitando los inconvenientes de la duplicación de infraestructuras sin ningún tipo de regulación. Es decir, se pueden disponer de tres o cuatro redes de fibra sin necesidad de que cada operador deba abrir costosas zanjas en las calles para desplegar su propia red de canalizaciones y conductos, pues ya utiliza los de Telefónica. Asimismo, un edificio, con una sola instalación de fibra, puede contar con la oferta de varios operadores.

Por otro lado, la resolución sobre mercados también estableció un marco claro para habilitar el cierre ordenado de centrales de la red de cobre de Telefónica cuando el área correspondiente a la central ya pueda ser atendida por la nueva red de fibra.

\subsection{El marco regulatorio adoptado por la CNMC en 2016}

Las medidas adoptadas en 2009 fueron efectivas: varios operadores desplegaron fibra utilizando la oferta regulada de conductos. No obstante, la red FTTH de Telefónica era, con diferencia, la red FTTH más extensa. Es decir, había zonas con varias redes NGA y otras que solo contaban con la red de Telefónica. 
Ante esta situación, el principal objetivo de la regulación de los mercados de banda ancha de 2016 fue lograr el equilibrio adecuado entre la promoción de la inversión eficiente y asegurar el mantenimiento de una competencia sostenible.

Para ello, y dado el éxito de la medida y su relevancia para el despliegue de redes FTTH, se mantuvieron las obligaciones de acceso a los conductos en todo el país. Asimismo, para evitar una disminución de la competencia en las zonas que no contasen con suficientes redes de fibra, la CNMC decidió realizar un novedoso análisis geográfico que contemplaba dos grandes zonas ${ }^{20}$ :

- Zona competitiva en redes de banda ancha ultrarrápida (Zona $B A U$ ), formada por 66 municipios, que incluyen la mayoría de grandes ciudades del país. En estos 66 municipios existían despliegues de red de nueva generación de al menos tres operadores, de tal manera que, de forma prospectiva, era esperable la competencia por parte de al menos tres operadores en la mayor parte del municipio. Estos municipios cubren el $35 \%$ de la población española.

- Zona no competitiva en redes de banda ancha ultrarrápida (Zona no BAU), formada por el resto del país. En esta zona, además del acceso a conductos, Telefónica debe proporcionar servicios mayoristas sobre su red de fibra, denominados NEBA local y NEBA fibra. Estos servicios deben tener la misma velocidad máxima que Telefónica ofrece

\footnotetext{
20 También se realizó un segundo nivel de segmentación que afecta a los servicios de acceso indirecto de banda ancha, por lo que en puridad existen cuatro zonas diferentes, pero por simplicidad se explica la segmentación principal que afecta a la obligación o no de prestar servicios regulados sobre fibra.
}

a sus clientes minoristas (actualmente $600 \mathrm{Mb} / \mathrm{s}$ ). Además, con el objeto de remunerar adecuadamente el riesgo en infraestructuras, Telefónica tiene libertad para fijar los precios mayoristas. La CNMC únicamente comprueba que dichos precios mayoristas superan un test de replicabilidad económica con los principales productos minoristas de Telefónica ${ }^{21}$.

Con el objeto de proporcionar un marco estable de certidumbre regulatoria en el que los operadores puedan planificar de forma adecuada sus inversiones, estas dos zonas se mantendrán estables hasta la próxima revisión del mercado de banda ancha ${ }^{22}$.

En cuanto a la eficacia del marco regulatorio de 2016, cabe señalar que a finales de 2018 los operadores han continuado desplegando redes FTTH y que además el nivel de competencia en el segmento NGA es incluso ligeramente superior al del global del mercado de banda ancha (Gráfico 4).

\section{El código de comunicaciones electrónicas. La conectividad como objetivo}

La Unión Europea aprobó, en diciembre de 2018, la revisión del marco normativo de las telecomunicaciones aplicable a toda Europa (el denominado Código Europeo de las Comunicaciones Electrónicas).

\footnotetext{
21 Este método de control de precios sigue la Recomendación de la Comisión Europea de 11 de septiembre de 2013 relativa a la coherencia en las obligaciones de no discriminación y en las metodologías de costes para promover la competencia y potenciar el entorno de la inversión en banda ancha (2013/466/UE).

22 El Código Europeo de Comunicaciones Electrónicas establece un plazo máximo de cinco años para revisar los mercados de comunicaciones electrónicas, con la posibilidad de solicitar una prórroga de un año adicional.
} 


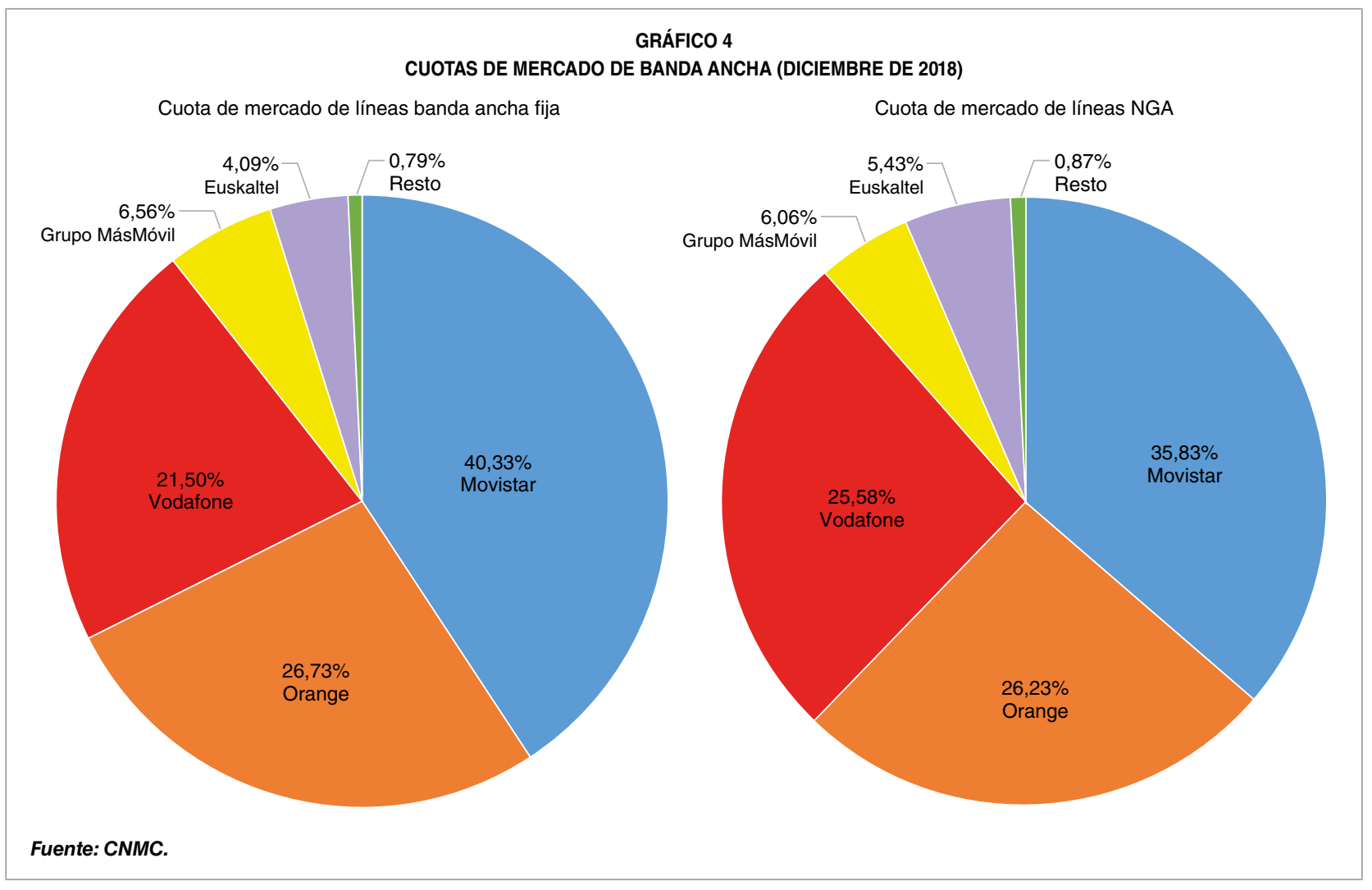

Este código incluye la adopción por parte de los consumidores de la conectividad a muy alta capacidad como objetivo regulatorio fundamental. De esta forma, aumenta el énfasis en la competencia en infraestructuras con reminiscencias de la solución española:

- Se da prioridad al acceso a infraestructuras pasivas (conductos, canalizaciones, postes) al imponer obligaciones al operador con poder significativo de mercado: esta medida está en vigor en España desde $2008^{23}$.

- Se refuerzan las posibilidades de imponer obligaciones simétricas a todos los operadores (en especial, la compartición de infraestructuras en el interior de

23 Si bien la decisión final de los mercados de banda ancha se adoptó en febrero de 2009 , la imposición de acceso a los conductos se avanzó en una medida cautelar aprobada por la CMT el 13 de noviembre de 2018. edificios) para favorecer los despliegues FTTH: esta medida está en vigor en España desde 2009.

- Las autoridades nacionales de regulación deberán realizar análisis de mercados geográficos más granulares que puedan resultar de la desregulación de algunas zonas con competencia en infraestructuras. Este es el enfoque adoptado por la CNMC en su revisión de mercados de 2016.

Es muy destacable que algunas de las principales medidas del código recientemente aprobado, que buscan su adopción en toda Europa, llevan años implementadas por el regulador español. El éxito en despliegues de red FTTH en España ha sido un factor decisivo para que el código incluya las medidas anteriores. 


\section{Conclusiones}

La calidad de las infraestructuras de telecomunicaciones es un factor clave para aumentar la competitividad del país. En las redes fijas, la arquitectura que permite las velocidades de transmisión más elevadas es la fibra hasta el hogar (Fiber-To-The-Home, FTTH).

España es un caso de éxito en los despliegues de fibra hasta el hogar, realizados además por varios operadores. Este éxito se explica por la existencia de amplios despliegues de redes de nueva generación (principalmente FTTH) realizados por varios operadores. La cobertura actual de redes NGA supera el $85 \%$, mientras que la cobertura prevista para 2020 podría superar el 90\%. Asimismo, España es el país de Europa con la penetración más elevada de la tecnología FTTH: un $44 \%$ de los hogares españoles ya cuenta con una conexión de fibra hasta el hogar frente al $10 \%$ de media en Europa.

Las medidas regulatorias adoptadas por CMT y CNMC han ayudado a fomentar estos despliegues, en especial la temprana imposición de acceso a los conductos de Telefónica y las obligaciones de acceso simétrico a las infraestructuras de fibra óptica instaladas en el interior de los edificios.

Por último, cabe destacar que el código de comunicaciones de la UE, aprobado en diciembre de 2018, tiene la conectividad a muy alta capacidad como objetivo prioritario y establece medidas reminiscentes de las soluciones adoptadas en España para impulsar los despliegues NGA.

\section{Bibliografía}

[1] Directiva (UE) 2018/1972 del Parlamento Europeo y del Consejo, de 11 de diciembre de 2018, por la que se establece el Código Europeo de las Comunicaciones Electrónicas. Recuperado de https://eur-lex.europa.eu/legal -content/ES/TXT/?uri=CELEX\%3A32018L 1972

[2] Informe de la Secretaría de Estado para el Avance Digital sobre la cobertura de banda ancha en España en el año 2018, publicado en abril de 2019. Recuperado de https://avancedigital.gob.es/banda-ancha/cobertura/ Documents/Cobertura-BA-2018.pdf

[3] Resolución de la CMT, de 22 de enero de 2009, por la que se aprueba la definición y el análisis del mercado de acceso (físico) al por mayor a infraestructura de red en una ubicación fija y el mercado de acceso de banda al por mayor, la designación de operador con poder significativo de mercado y la imposición de obligaciones específicas. Recuperado de https://www.cnmc.es/expedientes/mtz2008626

[4] Resolución de la CMT, de 12 de febrero de 2009, por la que se aprueba la imposición de obligaciones simétricas de acceso a los operadores de comunicaciones electrónicas en relación con las redes de fibra de su titularidad que desplieguen en el interior de los edificios. Recuperado de https://www.cnmc.es/ expedientes/mtz-2008965

[5] Resolución de la CNMC, de 24 de febrero de 2016, por la cual se aprueba la definición y el análisis del mercado de acceso local al por mayor facilitado en una ubicación fija y los mercados de acceso de banda ancha al por mayor, la designación de operadores con poder significativo de mercado y la imposición de obligaciones específicas. Recuperado de https://www.cnmc.es/expedientes/ anmedtsa215414 